\title{
Evaluation of cDNA Microarray Data by Multiple Clones Mapping to the Same Transcript
}

\author{
Dong Wang, ${ }^{1,2}$ Chenguang Wang, ${ }^{2}$ Lin Zhang,, Hui Xiao, ${ }^{1}$ Xiaopei Shen, Liping Ren, \\ Wenyuan Zhao, ${ }^{2}$ Guini Hong, Yuannv Zhang, ${ }^{2}$ Jing Zhu, Min Zhang, ${ }^{1}$ \\ Da Yang, Wencai $\mathrm{Ma}^{2}$, and Zheng Guo ${ }^{1,2}$
}

\begin{abstract}
Although novel technologies are rapidly emerging, the cDNA microarray data accumulated is still and will be an important source for bioinformatics and biological studies. Thus, the reliability and applicability of the cDNA microarray data warrants further evaluation. In cDNA microarrays, multiple clones are measured for a transcript, which can be exploited to evaluate the consistency of microarray data. We show that even for pairs of RCs, the average Pearson correlation coefficient of their measurements is not high. However, this low consistency could largely be explained by random noise signals for a fraction of unexpressed genes and/or low signalto-noise ratios for low abundance transcripts. Encouragingly, a large fraction of inconsistent data will be filtered out in the procedure of selecting differentially expressed genes (DEGs). Therefore, although cDNA microarray data are of low consistency, applications based on DEGs selections could still reach correct biological results, especially at the functional modules level.
\end{abstract}

\section{Introduction}

$\mathbf{T}$ HE CDNA-BASED MICROARRAY EXPERIMENTS are usually burdened with substantial amounts of noise, which may arise from nonspecific hybridization of the labeled samples to probes printed on the microarrays, print-tip effects, slide inhomogeneities, and variations in RNA isolation, purity, labeling and detection (Bilban et al., 2002). Among others, all these factors may introduce irreproducible measurements and thus low consistency of differentially expressed genes (DEGs) selected for a disease, which frequently raises doubts about the reliability of microarray applications (Ein-Dor et al., 2005; Frantz, 2005; Miklos and Maleszka, 2004). However, we recently demonstrated that the consistency between DEG lists detected from cDNA and oligo microarray data for a complex disease is high in the sense that different studies have actually detected similar results for the disease (Zhang et al., 2008). This result suggests that both cDNA and oligo microarray systems could capture true DEGs in complex diseases. Although more reliable data of gene expression profiles can be produced along with the rapid development of novel technologies, the cDNA microarray data accumulated in the past decades is still and will be an important source for various bioinformatics analyses and biological comparison studies.
Therefore, the reliability and applicability of the cDNA microarray data warrant careful evaluation.

Microarrays often yield measurements of multiple probe sets or clones representing the same transcript. For Affymetrix oligonucleotide microarrays, by analyzing the correlation of measurements of multiple probe sets representing the same transcript, studies have investigated the data quality influenced by probe designs and annotations (Chalifa-Caspi et al., 2004; Gautier et al., 2004; Harbig et al., 2005; Hwang et al., 2004; Nimgaonkar et al., 2003; Stalteri and Harrison, 2007; Zhang et al., 2005). For cDNA microarrays, it is also a common practice to measure multiple clones (sequence segments) for a transcript (Churchill, 2002), where the multiple clones can be classified into two types: different clones (referred to as DCs for short) for a transcript and replicate clones (referred to as RCs) for a transcript. Ideally, if a transcript is expressed in the microarrays, multiple clones representing the same transcript should have similar measurements, which can be exploited to evaluate the consistency of microarray data.

Here, using three cDNA microarray datasets, we show that the average Pearson correlation coefficient (PCC) of the measurements of DC pairs in each of the datasets is low, although it can be improved by updating clone annotations (Draghici et al., 2006). Even for pairs of RCs in each dataset,

\footnotetext{
${ }^{1}$ School of Life Science and Bioinformatics Centre, University of Electronic Science and Technology of China, Chengdu, 610054, People's Republic of China.

${ }^{2}$ School of Bioinformatics Science and Technology, Harbin Medical University, Harbin 150086, People's Republic of China.
} 
the average PCC of their measurements is only about 0.3 and above $12 \%$ of the pairs show negative correlations, which, however, could largely be explained by random noise signals for a fraction of unexpressed genes and/or low signal-to-noise ratios for low abundance transcripts in biological samples. On the other hand, in each dataset, over $80 \%$ of the RC pairs show positive correlations, indicating that cDNA microarrays can produce informative data for most of the expressed genes. More encouragingly, the procedure of selecting DEGs can statistically filter many inconsistent data. Indeed, most of the $\mathrm{RC}$ pairs with negative or low correlations will not be selected as DEGs in the three datasets. Therefore, despite the low data consistency for a fraction of the microarray data, applications based on DEG selections can still reach correct biological results especially at the functional modules level. This will increase our confidence on the reliability of many applications based on cDNA microarray data.

\section{Materials and Methods}

\section{cDNA microarray data and clone annotation}

The three large cDNA microarray datasets analyzed in this study are described in Table 1 . Clones were annotated to Unigenes (Dai et al., 2005; Mecham et al., 2004) through the SOURCE database in October 2006 (Uingene builder 196) and April 2008 (UinGene builder 211), respectively. There are two types of multiple clones: (1) DCs for a transcript which are different sequence parts for the same transcript, and (2) RCs for a transcript that are the same sequence part for the same transcript. Both DC and RC pairs for a transcript are technical replicates for measuring the expression of the transcript. Here, we assumed that each DC pair represents a single transcript as others usually do (Gautier et al., 2004). However, because current knowledge of alternative splicing for most genes is still far from complete (Dai et al., 2005), a DC pair according to current annotations might match to different alternative splicing products of a gene (Stalteri and Harrison, 2007), and thus the correlation of their measurements could be low. With the accumulation of the knowledge of alternative splicing, this problem could be gradually solved. As shown in Table 1, in the gastric (Chen et al., 2003), liver (Chen et al., 2004), and prostate (Lapointe et al., 2004) cancer datasets, respectively, the numbers of the UniGenes mapped with DCs decreased greatly from 22,731, 9,872 and 24,741 in October 2006 to 14,685, 6,137, and 16,412 in April 2008, whereas the numbers of the UniGenes mapped with RCs decreased slightly from $1,648,611$, and 1,472 to $1,554,575$, and 1,394 .

TABle 1. Three cDNA Datasets

\begin{tabular}{lrrr}
\hline & Gastric & Liver & Prostate \\
\hline Sample size (cancer vs. normal) & $103: 29$ & $82: 74$ & $62: 41$ \\
Total number of UniGenes $^{\text {a }}$ & 25,399 & 14,519 & 26,267 \\
& 20,757 & 12,932 & 21,341 \\
Number of DCs with UniGene $^{\text {a }}$ & 14,685 & 6,137 & 16,412 \\
& 22,731 & 9,872 & 24,741 \\
Number of RCs with UniGenes $^{\text {a }}$ & 1,554 & 575 & 1,394 \\
& 1,648 & 611 & 1,472 \\
\hline
\end{tabular}

${ }^{\mathrm{a}}$ The first and second lines represent the annotation results using SOURCE database in Apr. 2008 and Oct. 2006, respectively.

\section{Data preprocessing}

Data were preprocessed by logarithmic transformation and median normalization as adopted in the Oncomine database (Rhodes et al., 2007). The clones with missing rates above $20 \%$ were deleted and the other missing data were replaced by using the KNNimpute algorithm $(k=15)$ (Troyanskaya et al., 2001; Wang et al., 2006; Zhang et al., 2008). Because all the current normalization procedures are debatable (Do and Choi, 2006), we additionally tried LOWESS (Yang et al., 2002) and global median normalizations (Quackenbush, 2002) in our analysis. Similar results based on different normalization methods were observed (not shown in this note).

\section{Pearson correlation coefficient}

In each dataset, the PCC of the measurements of the DC and the RC pairs were calculated, respectively (Bergmann et al., 2004; Carter et al., 2004; Lee et al., 2003) and their distributions within different signal intensity ranges were analyzed. Here, the signal intensity of a clone in a dataset was defined as the average of the red and green intensity of the clone across all the samples in the dataset.

Similarly, as Carter et al. (2004) did, in each dataset, we estimated the random correlation distribution by independently permuting the sample labels of each clone and then computed the PCCs for all the clone pairs. The $p$-value of an observed correlation between two clones was calculated as the percentage of the permutated correlations exceeding the observed one. The FDR control procedure proposed by (Benjamini and Hochberg, 1995) was used to select clone pairs with significant correlation [false discovery rate (FDR) $<5 \%$ ].

\section{Selection of DEG}

For a UniGene measured by multiple clones, we used the average of the measurements of the multiple clones in a sample as the expression value of the UniGene in the sample (Breslin et al., 2005; Kapp et al., 2006). Then, in a dataset, DEGs were selected by SAM (Tusher et al., 2001), $t$-test (Cui and Churchill, 2003), and fold change (defined as the difference between the sample means of log-expressions) (Guo et al., 2006), respectively. The multiple tests were controlled by the FDR loosely defined as the expected percentage of false positives among the claimed DEGs (Benjamini and Hochberg, 1995). The distributions of the correlations for the DC and RC pairs included in the DEG lists selected from each dataset were analyzed respectively.

\section{Results}

\section{The measurement correlations for $D C$ and $R C$ pairs}

The distributions of the correlations for the DC pairs in the three cancer datasets are shown in Figure 1. In the gastric, liver, and prostate datasets, respectively, the average PCCs for the DC pairs were only $0.18,0.17$, and 0.18 when using the annotation data in October 2006, which increased to 0.32, 0.30, and 0.27 when using the updated annotation data in April 2008. In the three datasets respectively, 26, 29, and $26 \%$ of the DC pairs showed negative correlation when using the annotation data in October 2006 and the percentages decreased to $14.71,16.17$, and $16.94 \%$ when using the annotation data in April 2008. As shown in Figure 1, in each dataset, the PCCs of 

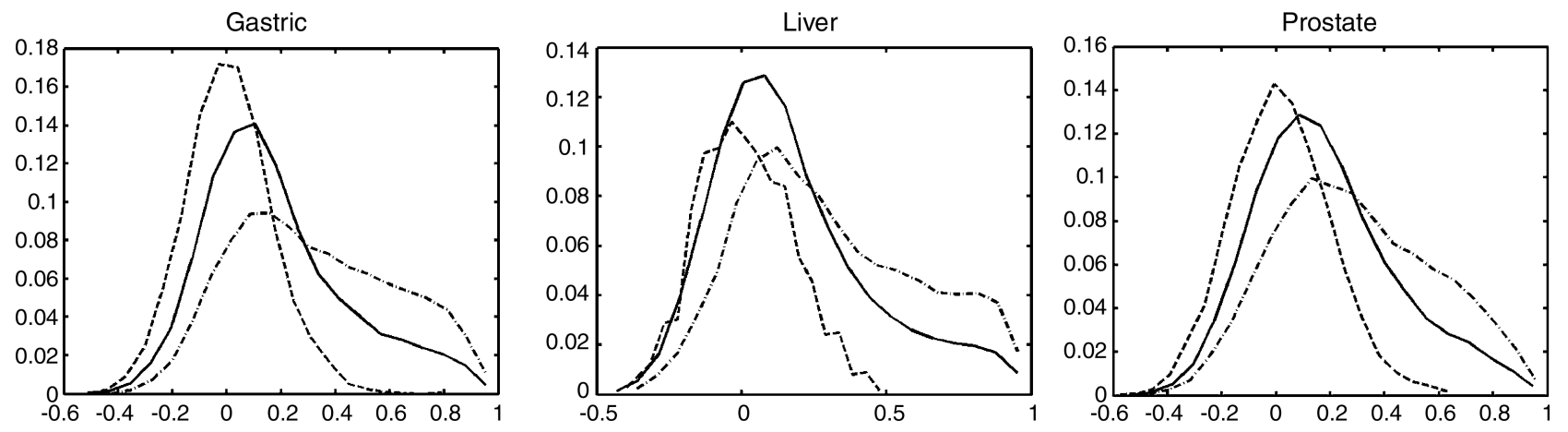

----.- Apr.2008 annotation - Oct.2006 annotation ---- Lost annotation

FIG. 1. Distributions of the measurement correlations for the DC pairs in the cancer datasets. The solid lines and dash dot lines represent the results based on the annotation data in October 2006 and April 2008, respectively. The dashed lines represent the results for the DC pairs whose annotation data in October 2006 were excluded in April 2008.

the DC pairs whose annotation data in October 2006 were excluded in April 2008 were symmetrically distributed around zero, indicating that the excluded clones might be wrongly annotated in October 2006. For example, in the liver dataset, four clones (IMAGE: 1160723, IMAGE: 117292, IMAGE: 201314, IMAGE: 81646) were mapped to the same UniGene Hs. 474596 in October 2006 but IMAGE: 201314 had been excluded in April 2008. The mean of the pairwise PCC setween IMAGE: 201314 and the other three clones was -0.003 , whereas the mean value of the left three clones was larger than 0.50 . Here, the result that the correlations of the measurements affected by annotation changes are symmetrically distributed around zero suggests that they might represent noise. Assuming the clones excluded in April 2008 did not represent any transcriptions, we could take these clones as technical replicates to estimate the noise levels in microarrays, as the average of the observed expression deviations of all these clones by a model proposed in Klebanov and Yakovlev (2007). In the three datasets, we could estimate the noise levels as $0.67,0.62$, and 0.57 respectively, which, however, could be overestimated because some clones excluded in April 2008 might represent multiple genes (Harbig et al., 2005; Stalteri and Harrison, 2007) and introduce biological variations. Thus, the noise levels estimated in such a way might be much higher than the true ones and thus could not be used to filter the rest of the clones.

The distributions of the correlations for the RC pairs in the three cancer datasets are shown in Figure 2. When using the annotation data in October 2006, the average PCCs for the RC pairs in the gastric, liver, and prostate datasets were still low as $0.29,0.32$, and 0.28 , respectively. When using the annotation data in April 2008, the results $(0.30,0.33$, and 0.27) were not improved. Specifically, the average PCCs of the RC pairs that were annotated in October 2006 but excluded in April 2008 were $0.26,0.34$, and 0.28 in the three datasets, showing no large difference from the results when using the annotation data in April 2008. This result suggests that the specificity of clone annotations has little influence on the measurement correlation of RC pairs. As shown in Figure 2, in the three datasets, respectively: $17.96,14.10$, and $16.03 \%$ of the RC pairs showed negative correlation when using the annotation data in October 2006, which were similar to the results (17.62, 12.54 , and $16.36 \%$ ) when using the annotation data in April 2008.

Obviously, when measurement variances exist, even the RC pairs may show low or negative correlation for transcripts with no or very low biological signals. As shown in Figure 3, in all the three datasets, the average PCCs for both the DC and
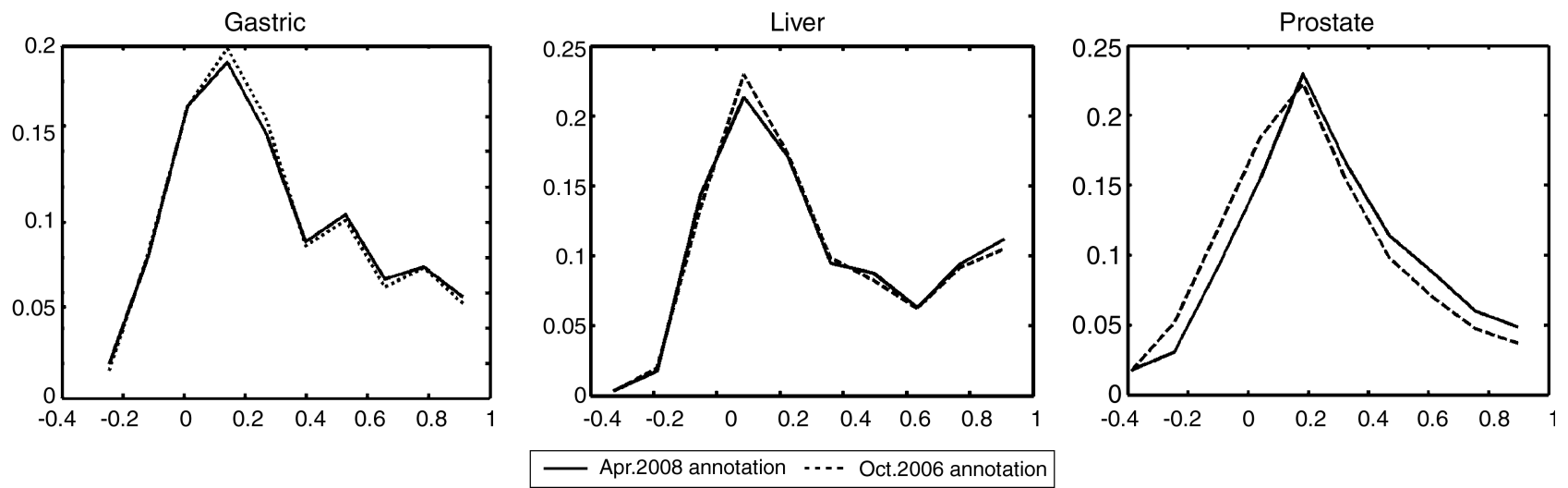

FIG. 2. Distributions of measurement correlations for the RC pairs in three datasets. The dash dot lines and solid lines represent the results based on the annotation data in October 2006 and in April 2008, respectively. In the three datasets, respectively, there are only 84, 31, and 76 RC pairs whose annotation data in October 2006 were excluded in April 2008 (correlation distributions are not shown). 

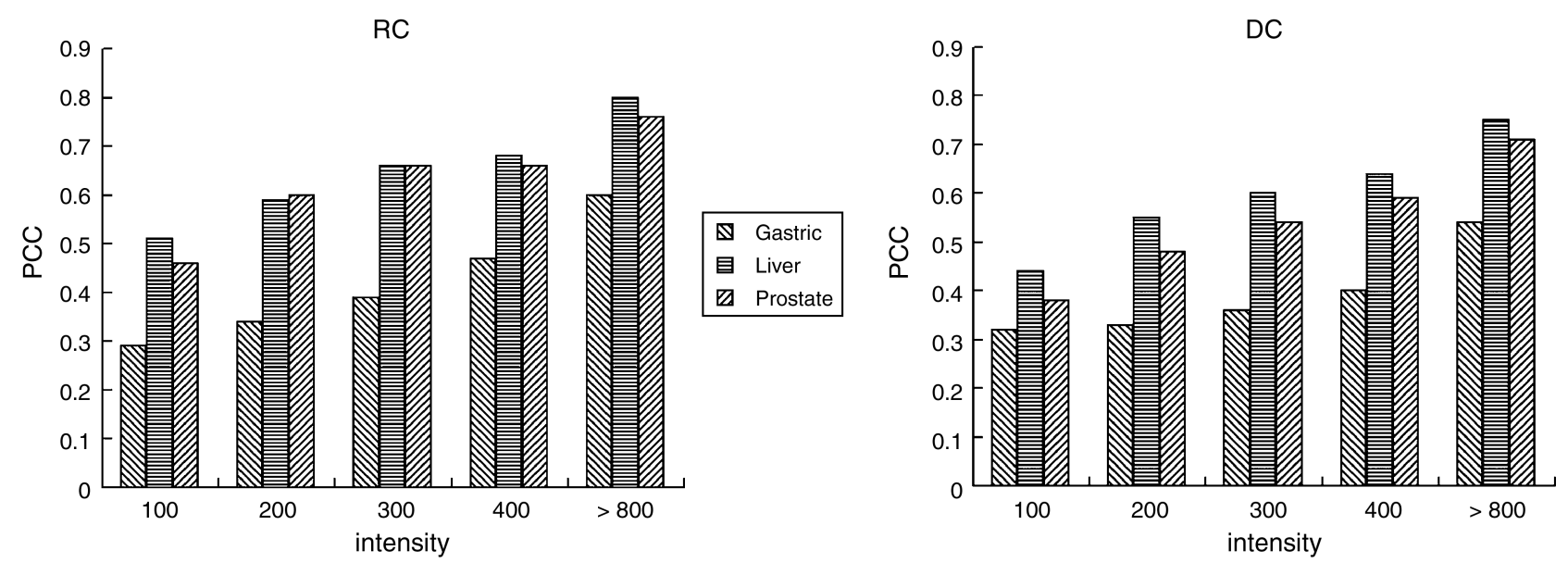

FIG. 3. Distributions of measurement correlations for the DC and RC pairs within different signal intensity ranges. The signal intensity of a clone in a dataset is defined as its average red and green intensity across all samples in the dataset.

RC pairs decreased as the signal intensity decreased. Therefore, the low average PCCs of the DC and RC pairs in a dataset could be a reflection of the complex biological reality that a high percentage of genes may not express or express at very low levels in tissue samples in a microarray experiment (Draghici et al., 2006; Shi et al., 2006). Intuitively, compared with the DCs, the RCs with exactly the same sequence should be more likely to have similar measurements because their hybridization specificity is certainly the same. However, in two of the three datasets, the DCs actually show a somewhat stronger correlation than the RCs. To explain this unexpected result, we compared the intensity values of the RC and DC pairs in the three datasets, respectively. As shown in Table 2, in both the gastric and prostate cancer datasets, the DC pairs have significantly higher intensity than the RC pairs. After DEG selection, this bias still existed. This bias could explain the results in these two datasets that the average PCC of the DC pairs are higher than those of the RC pairs and the negative correlation percentages of the DC pairs are lower than those of the RC pairs. In liver cancer dataset, the RC and DC pairs are not significantly different in intensity both before and after the DEG selection.

According to the annotation in April 2008, in the three datasets, the percentages of significant PCCs among all the negative PCCs for both the DC and RC pairs are zero. In

Table 2. The Average Intensity of the RC AND DC PAIRs IN THE Three DATAsETS

\begin{tabular}{lrrl}
\hline & $R C$ & $D C$ & $\mathrm{p}$-Value by t-Test \\
\hline ALL & & & \\
Gastric & 1,106 & 1,400 & 0.0000032 \\
Liver & 747 & 611 & 0.055 \\
Prostate & 273 & 442 & 0 \\
$D E G$ & & & \\
Gastric & 1,409 & 1,640 & 0.068 \\
Liver & 1,075 & 943 & 0.42 \\
Prostate & 304 & 559 & 0.00021 \\
\hline
\end{tabular}

RC, replicate clones; DC, different clones; DEG, differently expressed genes. contrast, the percentages of significant PCCs among all the positive PCCs for the DC pairs are 30.15, 34.73, and $19.12 \%$, and the values for the RC pairs are $17.75,30.96$, and $10.03 \%$. We note that many nonsignificant positive PCCs of the DC and RC pairs might be introduced by, especially for some lowly expressed genes, the inefficient sample sizes of the datasets and some others might indicate that current "new" annotations of clones may still contain errors.

\section{The DEG selection procedure as a filtering process for inconsistent data}

According to the annotation data in April 2008, we analyzed the correlation distribution of multiple clones mapping to the same transcripts selected as DEGs. In the gastric, liver, and prostate datasets, respectively, 6,467, 3,840, and 2,151 UniGenes were selected as DEGs by SAM at 0.01 FDR control level. In the three datasets respectively, there were 4,931, 1,770 , and 1,631 DC pairs corresponding to the selected DEGs. The average PCCs of these DC pairs increased to 0.41, 0.46, and 0.51 , and the percentages of these DC pairs showing negative correlations decreased to $8.87,7.64$, and $6.11 \%$, as shown in Figure 4 . The numbers of the RC pairs corresponding to the selected DEGs were 227, 86, and 68, among which the average PCCs increased to $0.36,0.51$, and 0.48 , and the percentages of the pairs with negative correlation decreased to $14.98,2.33$, and $7.35 \%$.

There were 735,308 , and 689 DC pairs corresponding to the top 1,000 DEGs selected from the three datasets, respectively. The average PCCs of these DC pairs increased to 0.54, 0.62, and 0.57 , whereas the percentages of the DC pairs with negative correlation decreased to $3.67,2.60$, and $4.50 \%$. For the 36 , 19 , and $42 \mathrm{RC}$ pairs from the top 1,000 DEGs in the three datasets, respectively, the average PCCs increased to 0.58 , 0.48 , and 0.55 , whereas the numbers of the pairs with negative correlation decreased to 2,1 , and 3 . Thus, a large fraction of the inconsistent clones were filtered out in the process of selecting DEGs. Similar results were observed when the DEGs were ranked and selected by $t$-test statistics or fold change, as shown in Figure 4.

Notably, in gastric cancer dataset, the DEG selection procedure filters a smaller fraction of negative correlation data. In 

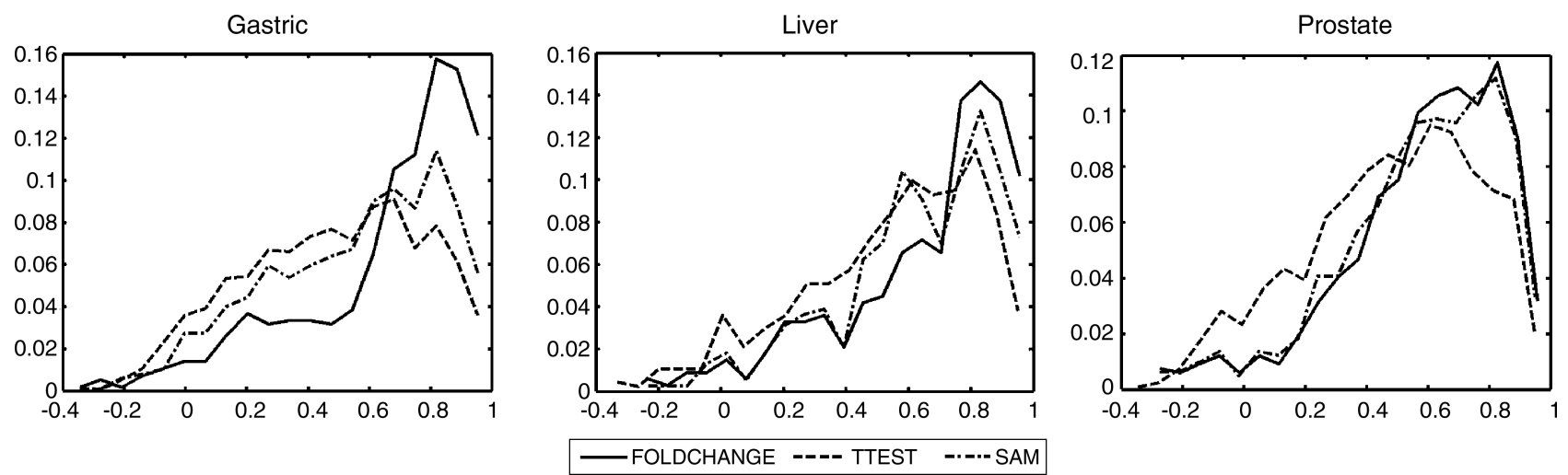

FIG. 4. Distributions of measurement correlations of the DC pairs corresponding to the top 1000 DEGs.

this dataset, there are only 29 normal samples, which may be insufficient to represent the characteristics of the normal population and reduce the efficiency of the detection of DEGs. Besides, in this dataset, the tumor sample size is more than three times over the normal sample size, and this sample imbalance could increase both the Type I error (false positives) and the Type II error (false negatives) for identifying DEGs (Yang et al., 2006). Thus, the DEG selection procedure in this dataset may be less efficient in filtering the inconsistent data.

\section{Discussion}

In each of the three cDNA datasets under this study, the average correlation of the measurements for either the DC or RC pairs mapping to the same UniGene was not high. For the DC pairs, the correlations can be improved by updating clone annotations. Thus, clones need to be carefully chosen to avoid ambiguous sequences, which may actually hybridize to transcripts from other genes (i.e., crosshybridization) or may be alternative splice variants of the same gene (Dai et al., 2005; Stalteri and Harrison, 2007; Tomiuk and Hofmann, 2001; Yu et al., 2007). However, even for the RC pairs, the average correlation of their measurements in each of the three datasets was not high and above $12 \%$ of these pairs showed negative correlations. This could possibly be produced by, besides the experimental errors mentioned in the Introduction, random noise signals for many unexpressed genes and/or low signalto-noise ratios for low abundance transcripts. It is known that only about $10-30 \%$ of the genes are expressed in a human cell (Li et al., 2002; Snijders et al., 2000), and thus about half of the human genes might be unexpressed or lowly expressed in tissue samples in a microarray experiment (Geschwind, 2000; $\mathrm{Su}$ et al., 2004). Obviously, only random noise signals are detectable for the clones representing the unexpressed genes, and thus their corresponding DC or RC pairs should show correlations randomly distributed around zero, supposing the background noise follows a normal distribution ( $\mathrm{Tu}$ et al., 2002). Because of the existence of a fraction of unexpressed genes or lowly unexpressed genes in biological samples, results should be interpreted carefully when using multiple clones mapping to the same transcript for evaluating the consistency of cDNA microarray data as well as for calibrating normalization protocols of microarray experiments (Fan and Niu, 2007; Fan et al., 2004; Lee et al., 2000; Smyth et al., 2005).
Notably, above $80 \%$ of the RC pairs in each dataset showed positive correlation, suggesting that the change directions of gene expressions can be reliably detected for the majority of expressed genes in a microarray experiment. Encouragingly, the DEG selection procedure can statistically filter a large fraction of inconsistent or noisy data. Thus, although many noises and measurement variations exist in microarray experiments, most DEGs selected from the cDNA microarray data could still be true discoveries. As recently demonstrated by us (Zhang et al., 2008), even when technical measurement variations are small, DEGs lists separately determined from current microarray studies for a complex disease may each capture only a small fraction of all the correlated molecular changes in the disease and thus be highly inconsistent. Fortunately, major functional modules significantly enriched with DEGs in a disease could be captured by only a small fraction of DEGs in the disease (Yang et al., 2008). Additionally, because functional modules for a disease are statistically determined by a set of DEGs, the results can become more robust to noisy data (Hosack et al., 2003; Yang et al., 2008). Therefore, although some uncertainty exists at the individual genes level, based on DEG selection in current cDNA microarray experiments, applications at functional modules level can still reach correct biological conclusions (Guo et al., 2006; Hosack et al., 2003; Subramanian et al., 2005; Zhu et al., 2007). Finally, we note that one important trend in microarray data analysis is to find significant functional categories by using continuous measures of gene expressions rather than preselecting DEGs (Breitling et al., 2004; Subramanian et al., 2005), which might be reasonable because the alterations of gene expressions should be continuous rather than discrete. However, lacking the process of selecting DEGs, such approaches might be less efficient in filtering noisy data, and thus their performances in studying noisy cDNA microarray data warrant our future studies.

\section{Acknowledgments}

This work was supported by the National Natural Science Foundation of China (Grant Nos. 30670539 and 30770558) and Scientific Research Fund of Heilongjiang Provincial Education Department (No. 11541156).

\section{Author Disclosure Statement}

The authors declare that no conflicting financial interests exist. 


\section{References}

Benjamini, Y., and Hochberg, Y. (1995). Controlling the false discovery rate: a practical and powerful approach to multiple testing. J R Stat Soc 57, 289-300.

Bergmann, S., Ihmels, J., and Barkai, N. (2004). Similarities and differences in genome-wide expression data of six organisms. PLoS Biol 2, E9.

Bilban, M., Buehler, L.K., Head, S., Desoye, G., and Quaranta, V. (2002). Defining signal thresholds in DNA microarrays: exemplary application for invasive cancer. BMC Genomics 3, 19.

Breitling, R., Amtmann, A., and Herzyk, P. (2004). Iterative Group Analysis (iGA): a simple tool to enhance sensitivity and facilitate interpretation of microarray experiments. BMC Bioinformatics 5, 34 .

Breslin, T., Krogh, M., Peterson, C., and Troein, C. (2005). Signal transduction pathway profiling of individual tumor samples. BMC Bioinformatics 6, 163.

Carter, S.L., Brechbuhler, C.M., Griffin, M., and Bond, A.T. (2004). Gene co-expression network topology provides a framework for molecular characterization of cellular state. Bioinformatics 20, 2242-2250.

Chalifa-Caspi, V., Yanai, I., Ophir, R., Rosen, N., Shmoish, M., Benjamin-Rodrig, H., et al. (2004). GeneAnnot: comprehensive two-way linking between oligonucleotide array probesets and GeneCards genes. Bioinformatics 20, 1457-1458.

Chen, X., Higgins, J., Cheung, S.T., Li, R., Mason, V., Montgomery, K., et al. (2004). Novel endothelial cell markers in hepatocellular carcinoma. Mod Pathol 17, 1198-1210.

Chen, X., Leung, S.Y., Yuen, S.T., Chu, K.M., Ji, J., Li, R., et al. (2003). Variation in gene expression patterns in human gastric cancers. Mol Biol Cell 14, 3208-3215.

Churchill, G.A. (2002). Fundamentals of experimental design for cDNA microarrays. Nat Genet 32(Suppl), 490-495.

Cui, X., and Churchill, G.A. (2003). Statistical tests for differential expression in cDNA microarray experiments. Genome Biol 4, 210.

Dai, M., Wang, P., Boyd, A.D., Kostov, G., Athey, B., Jones, E.G., et al. (2005). Evolving gene/transcript definitions significantly alter the interpretation of GeneChip data. Nucleic Acids Res 33, e175.

Do, J.H., and Choi, D.K. (2006). Normalization of microarray data: single-labeled and dual-labeled arrays. Mol Cells 22, 254-261.

Draghici, S., Khatri, P., Eklund, A.C., and Szallasi, Z. (2006). Reliability and reproducibility issues in DNA microarray measurements. Trends Genet 22, 101-109.

Ein-Dor, L., Kela, I., Getz, G., Givol, D., and Domany, E. (2005). Outcome signature genes in breast cancer: is there a unique set? Bioinformatics 21, 171-178.

Fan, J., and Niu, Y. (2007). Selection and validation of normalization methods for c-DNA microarrays using within-array replications. Bioinformatics 23, 2391-2398.

Fan, J., Tam, P., Woude, G.V., and Ren, Y. (2004). Normalization and analysis of cDNA microarrays using within-array replications applied to neuroblastoma cell response to a cytokine. Proc Natl Acad Sci USA 101, 1135-1140.

Frantz, S. (2005). An array of problems. Nat Rev Drug Discov 4, 362-363.

Gautier, L., Moller, M., Friis-Hansen, L., and Knudsen, S. (2004). Alternative mapping of probes to genes for Affymetrix chips. BMC Bioinformatics 5, 111.

Geschwind, D.H. (2000). Mice, microarrays, and the genetic diversity of the brain. Proc Natl Acad Sci USA 97, 10676-10678.
Guo, L., Lobenhofer, E.K., Wang, C., Shippy, R., Harris, S.C., Zhang, L., et al. (2006). Rat toxicogenomic study reveals analytical consistency across microarray platforms. Nat Biotechnol 24, 1162-1169.

Harbig, J., Sprinkle, R., and Enkemann, S.A. (2005). A sequencebased identification of the genes detected by probesets on the Affymetrix U133 plus 2.0 array. Nucleic Acids Res 33, e31.

Hosack, D.A., Dennis, G. Jr., Sherman, B.T., Lane, H.C., and Lempicki, R.A. (2003). Identifying biological themes within lists of genes with EASE. Genome Biol 4, R70.

Hwang, K.B., Kong, S.W., Greenberg, S.A., and Park, P.J. (2004). Combining gene expression data from different generations of oligonucleotide arrays. BMC Bioinformatics 5, 159.

Kapp, A.V., Jeffrey, S.S., Langerod, A., Borresen-Dale, A.L., Han, W., Noh, D.Y., et al. (2006). Discovery and validation of breast cancer subtypes. BMC Genomics 7, 231.

Klebanov, L., and Yakovlev, A. (2007). How high is the level of technical noise in microarray data? Biol Direct 2, 9.

Lapointe, J., Li, C., Higgins, J.P., van de Rijn, M., Bair, E., Montgomery, K., et al. (2004). Gene expression profiling identifies clinically relevant subtypes of prostate cancer. Proc Natl Acad Sci USA 101, 811-816.

Lee, J.K., Bussey, K.J., Gwadry, F.G., Reinhold, W., Riddick, G., Pelletier, S.L., et al. (2003). Comparing cDNA and oligonucleotide array data: concordance of gene expression across platforms for the NCI-60 cancer cells. Genome Biol 4, R82.

Lee, M.L., Kuo, F.C., Whitmore, G.A., and Sklar, J. (2000). Importance of replication in microarray gene expression studies: statistical methods and evidence from repetitive cDNA hybridizations. Proc Natl Acad Sci USA 97, 9834-9839.

Li, X., Gu, W., Mohan, S., and Baylink, D.J. (2002). DNA microarrays: their use and misuse. Microcirculation 9, 13-22.

Mecham, B.H., Klus, G.T., Strovel, J., Augustus, M., Byrne, D., Bozso, P., et al. (2004). Sequence-matched probes produce increased cross-platform consistency and more reproducible biological results in microarray-based gene expression measurements. Nucleic Acids Res 32, e74.

Miklos, G.L., and Maleszka, R. (2004). Microarray reality checks in the context of a complex disease. Nat Biotechnol 22, 615621.

Nimgaonkar, A., Sanoudou, D., Butte, A.J., Haslett, J.N., Kunkel, L.M., Beggs, A.H., et al. (2003). Reproducibility of gene expression across generations of Affymetrix microarrays. BMC Bioinformatics 4, 27.

Quackenbush, J. (2002). Microarray data normalization and transformation. Nat Genet 32(Suppl), 496-501.

Rhodes, D.R., Kalyana-Sundaram, S., Mahavisno, V., Varambally, R., Yu, J., Briggs, B.B., et al. (2007). Oncomine 3.0: genes, pathways, and networks in a collection of 18,000 cancer gene expression profiles. Neoplasia 9, 166-180.

Shi, L., Reid, L.H., Jones, W.D., Shippy, R., Warrington, J.A., Baker, S.C., et al. (2006). The MicroArray Quality Control (MAQC) project shows inter- and intraplatform reproducibility of gene expression measurements. Nat Biotechnol 24, 1151-1161.

Smyth, G.K., Michaud, J., and Scott, H.S. (2005). Use of withinarray replicate spots for assessing differential expression in microarray experiments. Bioinformatics 21, 2067-2075.

Snijders, A.M., Meijer, G.A., Brakenhoff, R.H., van den Brule, A.J., and van Diest, P.J. (2000). Microarray techniques in pathology: tool or toy? Mol Pathol 53, 289-294.

Stalteri, M.A., and Harrison, A.P. (2007). Interpretation of multiple probe sets mapping to the same gene in Affymetrix GeneChips. BMC Bioinformatics 8, 13. 
Su, A.I., Wiltshire, T., Batalov, S., Lapp, H., Ching, K.A., Block, D., et al. (2004). A gene atlas of the mouse and human proteinencoding transcriptomes. Proc Natl Acad Sci USA 101, 6062-6067.

Subramanian, A., Tamayo, P., Mootha, V.K., Mukherjee, S., Ebert, B.L., Gillette, M.A., et al. (2005). Gene set enrichment analysis: a knowledge-based approach for interpreting genome-wide expression profiles. Proc Natl Acad Sci USA 102, 15545-15550.

Tomiuk, S., and Hofmann, K. (2001). Microarray probe selection strategies. Brief Bioinform 2, 329-340.

Troyanskaya, O., Cantor, M., Sherlock, G., Brown, P., Hastie, T., Tibshirani, R., et al. (2001). Missing value estimation methods for DNA microarrays. Bioinformatics 17, 520-525.

Tu, Y., Stolovitzky, G., and Klein, U. (2002). Quantitative noise analysis for gene expression microarray experiments. Proc Natl Acad Sci USA 99, 14031-14036.

Tusher, V.G., Tibshirani, R., and Chu, G. (2001). Significance analysis of microarrays applied to the ionizing radiation response. Proc Natl Acad Sci USA 98, 5116-5121.

Wang, D., Lv, Y., Guo, Z., Li, X., Li, Y., Zhu, J., et al. (2006). Effects of replacing the unreliable cDNA microarray measurements on the disease classification based on gene expression profiles and functional modules. Bioinformatics 22, 2883-2889.

Yang, D., Li, Y., Xiao, H., Liu, Q., Zhang, M., Zhu, J., et al. (2008). Gaining confidence in biological interpretation of the microarray data: the functional consistence of the significant GO categories. Bioinformatics 24, 265-271.

Yang, K., Li, J., and Gao, H. (2006). The impact of sample imbalance on identifying differentially expressed genes. BMC Bioinformatics 7(Suppl 4), S8.
Yang, Y.H., Dudoit, S., Luu, P., Lin, D.M., Peng, V., Ngai, J., et al. (2002). Normalization for cDNA microarray data: a robust composite method addressing single and multiple slide systematic variation. Nucleic Acids Res 30, e15.

Yu, H., Wang, F., Tu, K., Xie, L., Li, Y.Y., and Li, Y.X. (2007). Transcript-level annotation of Affymetrix probesets improves the interpretation of gene expression data. BMC Bioinformatics 8, 194.

Zhang, J., Finney, R.P., Clifford, R.J., Derr, L.K., and Buetow, K.H. (2005). Detecting false expression signals in high-density oligonucleotide arrays by an in silico approach. Genomics 85, 297-308.

Zhang, M., Yao, C., Guo, Z., Zou, J., Zhang, L., Xiao, H., et al. (2008). Apparently low reproducibility of true differential expression discoveries in microarray studies. Bioinformatics 24, 2057-2063.

Zhu, J., Wang, J., Guo, Z., Zhang, M., Yang, D., Li, Y., et al. (2007). GO-2D: identifying 2-dimensional cellular-localized functional modules in Gene Ontology. BMC Genomics 8, 30.

Address correspondence to: Zheng Guo

Bioinformatics Centre

School of Life Science

University of Electronic Science and Technology of China

Chengdu 610054, P.R. China

E-mail: guoz@ems.hrbmu.edu.cn 


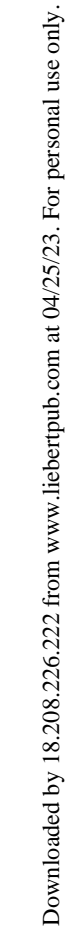

